\title{
Spinal cord injury complicating a thoracolumbar selective nerve root block in a deformed spine: neurological and functional outcome
}

\author{
N Kumar
}

Study design: A case report of a female patient who sustained injury to the thoracic spinal cord as a direct result of thoracolumbar selective nerve root block.

Objective: To raise the awareness that selective nerve root infiltration, a very common procedure in a pre-existing deformity, may cause injury to the spinal cord with dire consequences, albeit rarely.

Case report: An 82-year-old retired nurse (who had sustained osteoporotic compression fractures of the T12 vertebra) who presented with a 2-year history of continuous low back pain following a fall into a pothole. Immediately after image-guided, transforaminal infiltration of left side T12 root the patient developed sudden onset of complete motor and sensory T10 level flaccid paraplegia. No abnormality was noted on T2 magnetic resonance imaging (MRI) of the spine on the same day. Eleven days later, T2 MRI of the spine with gadolinium contrast revealed an increased fluid signal in conus medullaris. At 2-year follow-up, the neurological improvement has been good (L2 level American Spinal Injury Association/International Spinal Cord Society (ASIA/ISCoS) neurological standard scale (AIS) D paraplegia), urinary and sensory disorders are still present.

Conclusion: Injury to the spinal cord is known to occur in interventions such as epidural steroid infiltrations. Over time, pain management in relation to the new and emerging interventions on spine have changed. What might not be appreciated is the awareness that such interventions may cause injury to the spinal cord with serious consequences. Despite potential occurrence, in the case reported here, the neurological and functional prognosis is good with an expert, early and appropriate management in a spinal injuries centre.

Spinal Cord (2015) 53, S3-S5; doi:10.1038/sc.2014.180

\section{INTRODUCTION}

Although iatrogenic spinal cord paralysis has been described after inadvertent intrathecal injection, a medium and long-term neurological and functional outcome based on validated international standards has not been previously described as a sequelae of an image-guided, transforaminal infiltration block of T12 root in preexisting deformed spine. Here, a rare complication with selective block in thoracolumbar spine with lesions in the spinal cord with a good outcome following an expert, early and appropriate management in a spinal injuries centre with 2 years follow-up has been described.

\section{CASE REPORT}

An 82-year-old woman, a retired nurse, presented with a 2-year history of low back pain and left-sided T12 radicular pain following a fall into a pothole. She was diagnosed to have osteoporotic compression fracture of the T12 vertebra. Preliminary clinical examination in pain management clinic revealed localised tenderness over T12. The neurological examination was normal. She had tried a variety of analgesics including amitriptyline and pregabalin with no benefit.

Following discussion, she consented for an elective image-guided, transforaminal infiltration block of left T12 root. With the patient in a prone position in the operating theatre, under image guidance, a 22-gauge needle was introduced in the left transforaminal region close to T12 root. There was no cerebrospinal fluid or blood on aspiration. Under image intensifier control, needle position was confirmed and positive epidurogram was obtained with $0.5 \mathrm{ml}$ Omnipaque 240 (Figure 1). Immediately after the transforaminal infiltration of the left side $\mathrm{T} 12$ root with $80 \mathrm{mg}$ of dexamethasone and $10 \mathrm{ml}$ of $1 \%$ lignocaine, the patient developed sudden onset of complete motor and sensory T10 level flaccid paraplegia. Neurology improved from complete paraplegia to incomplete paraplegia. She also had incontinence of both bowel and bladder and no sensation of bladder fullness. No abnormality was noted in the same day T2 MRI of the spine.

Four days later she was admitted to the Midlands Centre for spinal Injuries (MCSI). Neurologic examinations were conducted according to the American Spinal Injury Association/International Spinal Cord Society (ASIA/ISCoS) neurological standard scale (AIS) by trained doctors at the MCSI. She had T10 level AIS-C paraplegia (Table 1).

Eleven days later, axial images of T2 MRI of the spine with gadolinium contrast revealed an increased fluid signal in the conus medullaris (Figure 2). These changes persisted in repeated MRI at 8 weeks (Figure 3 ). Sagittal T2-weighted MRI were negative at 11 days and at 8 weeks. 


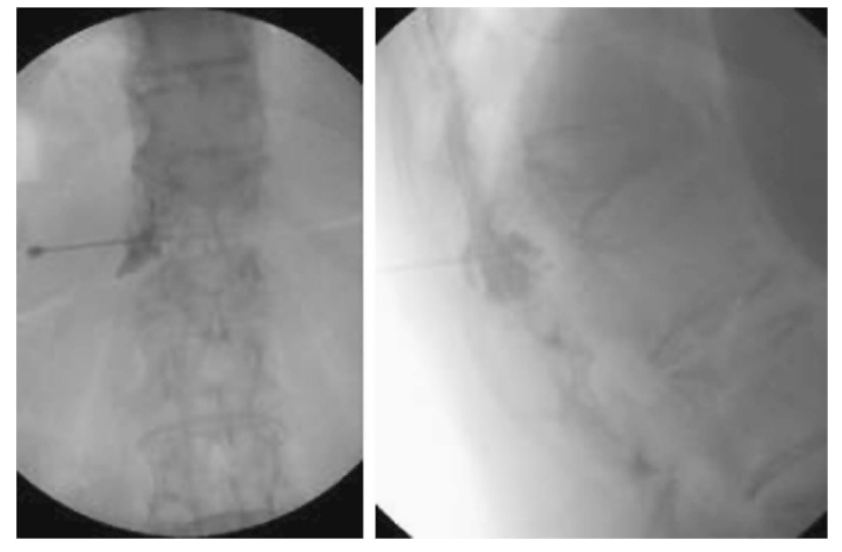

Figure 1 Under image guidance, a 22-gauge needle was introduced into the left transforaminal region close to T12 root, needle position was confirmed and positive epidurogram was obtained with Omnipaque 240.

\section{Table 1 AIS outcome}

\begin{tabular}{lccc}
\hline & Admission to MCSI & At 6 weeks & At 2 years \\
\hline ASIA motor score & 80 & 93 & 97 \\
ASIA pin prick score & 102 & 110 & 110 \\
ASIA light touch score & 111 & 111 & 113 \\
AIS & $\mathrm{C}$ & $\mathrm{D}$ & $\mathrm{D}$ \\
\hline
\end{tabular}

Abbreviations: ASIA, American Spinal Injury Association; AIS, American Spinal Injury Association/International Spinal Cord Society (ASIA/ISCoS) neurological standard scale; MCSI, Midlands Centre for Spinal Injuries.

Following bed rest for 3 weeks she improved to L2 level AIS D paraplegia (Table 1).

She participated in a structured, evidence-based, goal-oriented rehabilitation programme at the MCSI. Rehabilitation was measured using the patient notes, Needs Assessment Checklist (Figure 4), fortnightly held goal planning meeting sheets and Spinal Cord Independence Measure version III scores, which were collected by multidisciplinary team in goal planning meetings.

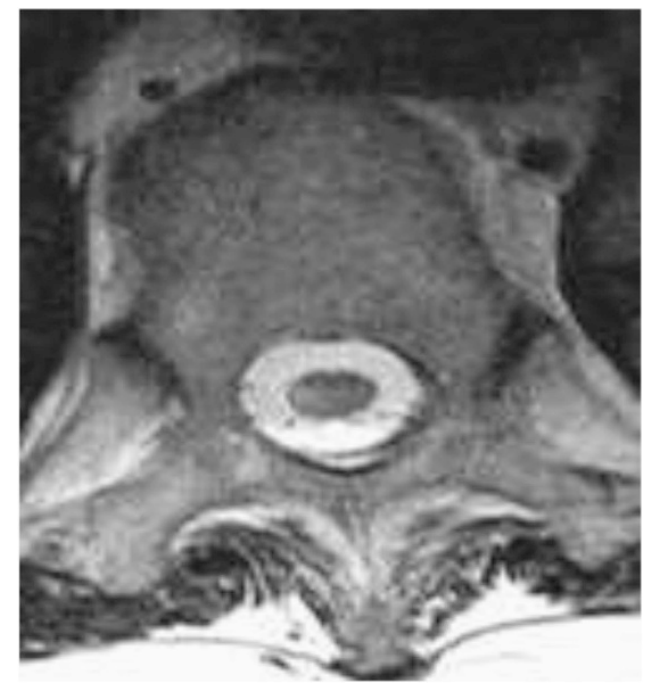

Figure 2 Eleven days later, upon T2 MRI of the spine with gadolinium contrast, an increased fluid signal in the distal cord was noted in axial image only, suggesting ischaemia.

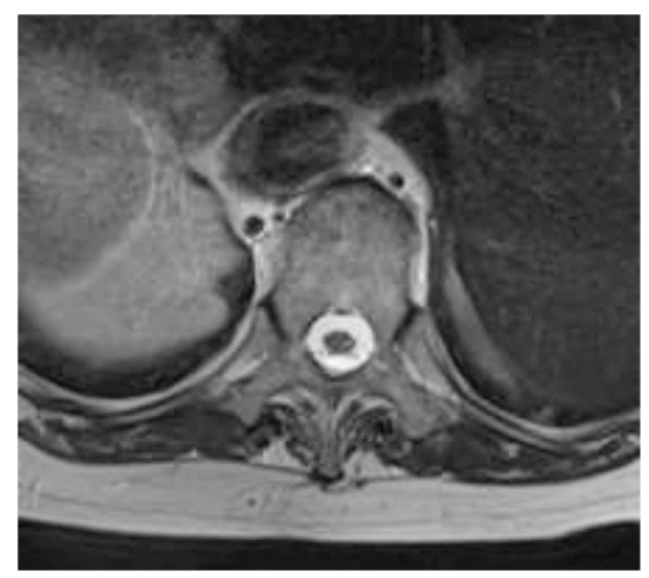

Figure 3 At 8 weeks, very subtle signal change in the lower spinal cord was noted on the axial T2 MRI of the spine.

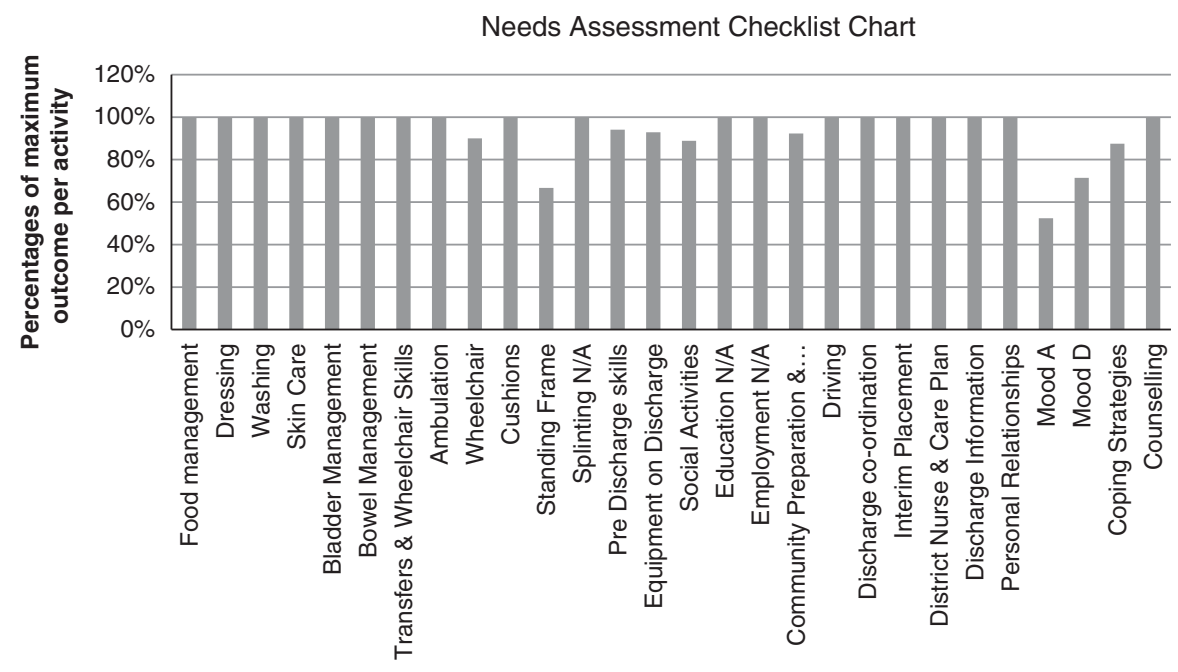

Rehabilitation Activities

Figure 4 Rehabilitation outcome based on Needs Assessment Checklist Chart-the results are presented as percentages of maximum outcome per activity at completion of rehabilitation programme. 


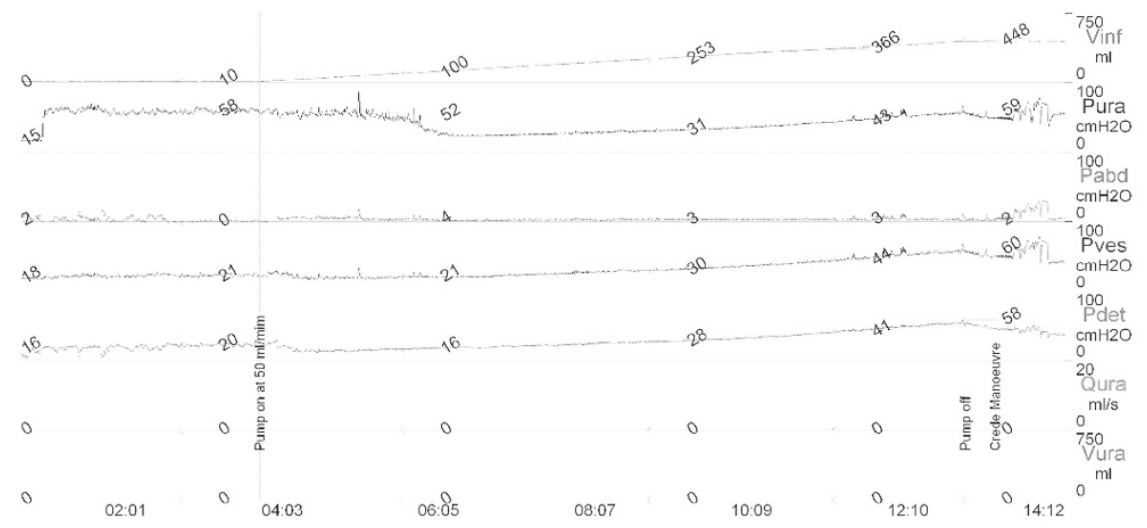

Figure 5 Videourodynamic analysis at 3 months.

Table 2 Spinal Cord Independence Measure (SCIM III) at 1 week after mobilisation and 2 years follow-up

\begin{tabular}{lcc}
\hline & 1 week & \\
& 12 & Atter 2 years \\
\hline Subscale self-care & 19 & 18 \\
Subscale respiration and sphincter & & 33 \\
management & 8 & \\
Subscale mobility & 39 & 76 \\
SCIM III (total score) & & 25 \\
\hline
\end{tabular}

She started mobilising in a wheelchair. Sphincter dysfunction was still present. A videourodynamic study at 3 months (Figure 5) revealed, absent bladder sensation during filling cystometry, underactive detrusor function, low compliance during filling cytometry and non-relaxing urethral function during voiding. The patient lacked spontaneous micturition and performed four intermittent selfcatheterisations daily, with no urine leakage.

At 2-year follow-up, neurological (Table 1) and functional (Table 2) improvements have been good.

\section{DISCUSSION}

Although review of literature identified similar cases following epidural infiltration, this is a rare case report of paraplegia following thoracolumbar selective nerve root infiltration in a previously deformed spine. Most authors suggest ischaemia caused by damage to medullary artery, arterial spasm or corticosteroid-induced occlusion could result in medullary infarction. ${ }^{1,2}$

The pre-existing fracture with deformity could have been a predisposing factor contributing to the intrinsic damage to the spinal cord during the intervention.

This serious incident should prompt us all to focus attention to anatomical changes to the intervertebral foramen and interference to the spinal cord blood supply at the foramen level. ${ }^{3}$

Owing to the relative paucity of comparative studies and lack of credible evidence, superiority of one method of injection therapy in low back pain over the other, patients should be encouraged to make an informed choice. ${ }^{4,5}$

\section{CONCLUSION}

Injury to the spinal cord is known to occur in interventions such as epidural steroid infiltrations. Over time, pain management in relation to the new and emerging interventions on spine have changed. What might not be appreciated is the awareness that such interventions may cause injury to the spinal cord with serious consequences. Patients should be encouraged to make an informed choice with information about such serious risks at the time of consent. Despite potential occurrence, in the case reported here, the neurological and functional prognosis is good with an expert, early and appropriate management in a spinal injuries centre.

\section{CONFLICT OF INTEREST}

The author declares no conflict of interest.

\section{ACKNOWLEDGEMENTS}

I thank Professor W El Masri, Mr A Osman, Mr J R Chowdhury, Mr S Bandi and other medical staff, all the nurses, psychologists, occupational and physiotherapists for their support in enabling the collection of the data without which the publication of this paper would not have been possible.

1 Thefenne L, Dubecq C, Zing E, Rogez D, Soula M, Escobar E et al. A rare case of paraplegia complicating a lumbar epidural infiltration. Ann Phys Rehabil Med 2010; 53: 575-583.

2 Houten JK, Errico TJ. Paraplegia after lumbosacral nerve root block: report of three cases. Spine J 2002; 2: 70-75.

3 Ram S, Osman A, Cassar-Pullicino VN, Short DJ, Masry WE. Spinal cord infarction secondary to intervertebral foraminal disease. Spinal Cord 2004; 42: 481-484.

4 Staal JB, de Bie R, de Vet HCW, Hildebrandt J, Nelemans P. Injection therapy for subacute and chronic low-back pain. Cochrane Database of Systematic Reviews 2008, Issue 3. Art. No: CD001824.

5 Chang-Chien GC, Knezevic NN, McCormick Z, Chu SK, Trescot AM, Candido KD et al. Transforaminal versus interlaminar approaches to epidural steroid injections: a systematic review of comparative studies for lumbosacral radicular pain. Pain Physician 2014; 17: E509-E524. 